Pacific Journal of Mathematics

ON THE HYPERPLANE SECTION THROUGH A RATIONAL 


\section{ON THE HYPERPLANE SECTION THROUGH A RATIONAL POINT OF AN ALGEBRAIC VARIETY}

\section{WEI-EIHN KUAN}

Let $V / k$ be an irreducible affine algebraic variety of dimension $\geqq 3$ defined over an infinite field $k$ with $\mathfrak{p}$ as its prime ideal in $k\left[X_{1}, \cdots, X_{n}\right]$. Let $P$ be a rational normal point on $V / k$. It is proved that (1) for a generic hyperplane $H_{u}$ through $P,\left(\mathfrak{p}, H_{u}\right)$ is a prime ideal and $\left(\mathfrak{p}, H_{u}\right)$ is quasi-absolutely (absolutely irreducible) if $\mathfrak{p}$ is quasi-absolutely (absolutely irreducible). (2) It is not true in general that $V \cap H_{u}$ is normal at $P$; however, $V \cap H_{u}$ is normal at $P$ if the local ring of $V / k$ at $P$ is also Cohen-Macaulay (Theorem 8).

It is well known [11] that if $V / k$ is a normal variety of dimension $\geqq 2$, then for almost all hyperplanes $H$ the section $V \cap H$ is again a normal variety. This research is motivated by this result to study the following problem: If $V / k$ is normal at a rational point $P$ on $V$, will hyperplane sections of $V$ through $P$ be normal at $P$ ? Section 1 localizes some of the results of [11]. Section 2 describes the ideal decomposition of the generic hyperplane section through a given rational point of an irreducible variety, and Section 3 gives a negative answer to the problem of normality. As a consequence the converse of [3; Lemma 4, p. 360] is invalid in general.

1. Generalities. In the following and the subsequent sections, a variety $V / k$ shall mean an irreducible algebraic variety in the affine space $A^{n}$ defined over a field $k$ of arbitrary characteristic.

Recall the following definitions.

Definition 1. Let $V / k$ be a variety with $(\xi)=\left(\xi_{1}, \cdots, \xi_{n}\right)$ as a generic point over $k$, and let $P$ be a point on $V$. Let

$$
k[\xi]_{p}=\left\{\frac{f(\xi)}{g(\xi)} \mid f, g \in k[\xi] \text { and } g(P) \neq 0\right\}
$$

be the local ring of $V$ at $P$ in the function field $k(\xi)$ of $V$ over $k$. We say that $P$ is $k$-normal on $V$ if $k[\xi]_{p}$ is integrally closed in $k(\xi)$, that $P$ is $k$-simple on $V$ if $k[\xi]_{p}$ is a regular local ring, and that $P$ is singular on $V$ if $P$ is not $k$-simple on $V$.

Definition 2. Let $V / k$ be a variety of dimension $r$, and let $P$ be a point on $V$. We say that $V / k$ is locally free of $s$-dimensional 
singularities at $P$ if every $s$-dimensional subvariety of $V$ containing $P$ is $k$-simple on $V$.

DEFINITION 3. Let $R$ be a finite integral domain $k\left[\xi_{1}, \cdots, \xi_{n}\right]$ over a field $k$ or a localization thereof relative to a prime ideal of $k\left[\xi_{1}, \cdots, \xi_{n}\right]$. Let $\mathfrak{p}$ be a prime ideal of $R$ we define

ht $\mathfrak{p}=$ max. (length of chains of prime ideals contained in $\mathfrak{p}$ ), depth $\mathfrak{p}=\max$. (length of chains of prime ideals containing $\mathfrak{p}$ ), $\operatorname{dim} \mathfrak{p}=$ transcendence degree of the quotient field of $R / \mathfrak{p}$ over $k$, $\operatorname{dim} R=$ transcendence degree of the quotient field of $R$ over $k$. It is well known that $h t \mathfrak{p}+\operatorname{depth} \mathfrak{p}=\operatorname{dim} R$ and $\operatorname{dim} \mathfrak{p}=\operatorname{depth} \mathfrak{p}$.

The following criterion for local normality is parallel to [11; Th. 3, p. 363] and is well known [8; (12.9), p. 41].

Proposition 1. Let $V / k$ be a variety of dimension $r$ defined over a field $k$, and let $P$ be a point of dimension $s$ on $V . P$ is k-normal on $V$ if and only if (1) $V / k$ is locally free of $(r-1)$-dimensional singularities at $P,(2)$ every nonzero principal ideal $(a) \cdot k[\xi]_{p}$ is unmixed of dimension $r-s-1$.

Proposition 2. Let $V / k,(\xi)$, and $P$ be the same as those in Proposition 1 , let $k[\xi]_{p}^{*}$ be the integral closure of $k[\xi]_{p}$, and let $\mathfrak{S}_{p}$ be the conductor of $k[\xi]_{p}$. If $V$ is locally free of $(r-1)$-dimensional singularities at $P$ and if $\mathfrak{F}_{p} \neq(1)$, then every nonzero element of $\mathfrak{F}_{p}$ generates a mixed principal ideal.

Proof. Let $\alpha \in k[\xi]_{p}^{*}$ not in $k[\xi]_{p}$, and let $c \in \mathfrak{S}_{p}$, whence $c \alpha \in k[\xi]_{p}$, say $c \alpha=b, b \in k[\xi]_{p}$. Then $(c) \cdot k[\xi]_{p}$ must be mixed. Indeed, if $(c) k[\xi]_{p}$ were unmixed, and let $\mathfrak{p}_{1}, \cdots, \mathfrak{p}_{t}$ be the associated prime ideals of (c) $k[\xi]_{p}$, then $\operatorname{dim} \mathfrak{p}_{i}=r-s-1$, for $i=1,2, \cdots, t$. $\alpha$ is integral over $k[\xi]_{p}$, hence integral over $\left(k[\xi]_{p}\right)_{\mathfrak{p}_{i}}$ for $i=1,2, \cdots, t$. By hypothesis $\left(k[\xi]_{p}\right)_{\mathfrak{p}_{i}}$ is a regular local ring of dimension 1 , for $i=1,2, \cdots, t$, therefore $\left(k[\xi]_{p}\right)_{\mathfrak{p}_{i}}$ is integrally closed for $i=1,2, \cdots, t$. Hence $\alpha \epsilon$ $\bigcap_{i=1}^{t}\left(k[\xi]_{p}\right)_{\mathfrak{p}_{i}}$ and $b \in\left(\bigcap_{i=1}^{t}(c)\left(k[\xi]_{p}\right)_{\mathfrak{p}_{i}}\right) \cap k[\xi]_{p}=\bigcap_{i=1}^{t} \mathfrak{q}_{i}$, where $\mathfrak{q}_{1} \cap \cdots \cap \mathfrak{q}_{t}$ is a primary decomposition of $(c) k[\xi]_{p}$. Thus $b \in(c) b[\xi]_{p}$, i.e., $\alpha \in k[\xi]_{p}$, a contradiction.

Let $V / k$ be a variety of dimension $r$ defined over a field $k$ with

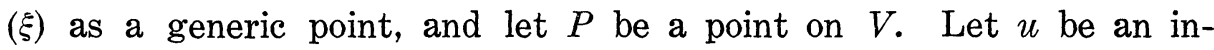
determinate over $k(\xi)$, it is well known that $V$ is a variety over $k(u)$ with $(\xi)$ as a generic point of $V$ over the pure transcendental extension field $k(u)$. Let $k(u)[\xi]_{p}=\{f(u ; \xi) / g(u ; \xi) \mid f, g \in k(u)[\xi]$ and $g(u ; p) \neq 0\}$ 
be the local ring of $V$ at $P$ over $k(u)$. We have, by [10, (d), p. 64], the following lemma.

LEMma 1. $k[\xi]_{p}$ is integrally closed if and only if $k(u)[\xi]_{p}$ is integrally closed.

Recall the definition of the ground form of an unmixed $r$-dimensional ideal $\mathfrak{U}^{\prime},[11 ; \mathrm{p} .373]$, as following: Let $\mathfrak{i}$ be an unmixed $r$-dimensional ideal in the polynomial ring $k\left[X_{1}, \cdots, X_{n}\right]$, we form $r+1$ linear forms in the $X_{i}$ 's with indeterminates coefficients $u_{i j}: z_{i}=u_{i 1} x_{1}+\cdots+u_{i n} X_{n}$, $i=1,2, \cdots, r+1$, and consider the ideal $\mathfrak{I} \cdot k(u)[X] \cap k(u)\left[z_{1}, \cdots, z_{r-1}\right]$, where $k(u)[X]=k\left(u_{11}, \cdots u_{r+1 n}\right)\left[X_{1}, \cdots, X_{n}\right]$, which is a principal ideal $\left(E\left(z_{1}, \cdots, z_{r+1} ; u\right)\right)$ in $k(u)[X]$. If $E$ is normalized so as to be a polynomial in the $u_{i j}$ and primitive in them, so that $E$ is defined to within a factor in $k$, then $E$ is the elementary divisor form or the ground form of 2 . The polynomial $E$ is integral in any $z_{i}$ over the other $z_{i}$ 's and is a polynomial in $z_{1}, \cdots, z_{r+1}$ of least degree in $z_{r+1}$, which is in $\mathfrak{U} \cdot k(u)[X]$. If $\mathfrak{U}$ is prime, then its ground form is irreducible, the converse is not true in general; but $\mathfrak{A}$ is primary if and only if its ground form is a power of an irreducible polynomial [9; Th. 9, p. 252]. $\mathfrak{Y}$ is prime and absolutely irreducible if and only if $(E)$ is prime and absolutely irreducible [9; Th. 15, p. 259]. If $\mathfrak{X}$ is prime and quasiabsolutely irreducible, then $(E)$ is prime and quasi-irreducible $[11, \mathrm{p}$. 373].

Proposition 3. Let $V / k$ be an r-dimensional variety defined over a field $k$ with $\mathfrak{p}$ as its prime ideal in $k[X]\left(=k\left[X_{1}, \cdots, X_{n}\right]\right)$. Let $p$ be a point on $V$ and let $E$ be the ground form of $\mathfrak{p}$. Then $V$ is $k$ normal at $p$ if and only if $\left(\mathfrak{p}, \partial E / \partial z_{r+1}\right) \cdot k(u)[X]_{p}$ is unmixed.

Proof. By Lemma $1, V$ is $k$-normal at $P$ if and only if $V$ is $k(u)$-normal at $P$. By [13; Lemma 2, p. 132] $V / k(u)$ is free of $(r-1)$ dimensional singularities at $P$. Let $(\xi)$ be a generic point of $V / k(u)$, and pass to $k(u)[\xi]$, we assert that $k(u)[\xi]_{p}$ is integrally closed if and only if $\left(\partial \bar{E} / \partial \bar{z}_{r+1}\right) \cdot k(u)[\xi]_{p}$ is unmixed, where the bar denotes residue. By the proof of [11; Th. 5, p. 365], we have $\partial \bar{E} / \partial \bar{z}_{r+1} \in \mathfrak{E}$, the conductor of $k(u)[\xi]$ in its integral closure $k(u)[\xi]^{*}$. Let $\mathfrak{S}_{p}$ be the conductor of $k(u)[\xi]_{p}$ in its integral closure $k(u)[\xi]_{p}^{*}$. By [15; Lemma, p. 269], (5 . $k(u)[\xi]_{p}=\mathfrak{\complement}_{p}$. Therefore $\partial \bar{E} / \partial \bar{z}_{r+1} \in \mathfrak{\complement}_{p}$. By Proposition 2, we have that $k(u)[\xi]_{p}$ is integrally closed if and only if $\left(\partial \bar{E} / \partial \bar{z}_{r+1}\right) \cdot k(u)[\xi]_{p}$ is unmixed.

2. Irreducibility of generic hyperplane section through a normal point. Let $V / k$ be a variety of dimension $r \geqq 2$. Let $P \in V$ be a rational point. We are studying the generic hyperplane section 
of $V$ through $P$. Without loss of generality, we may assume once for all in the sequel that $V$ passes through (0) the origin of the affine space and that $P=(0)$. We shall denote the prime ideal of $V / k$ by $\mathfrak{p}$ in the sequel. Let $u_{1}, \cdots, u_{n}$ be $n$ indeterminates over $k$, and let $H_{u}$ be the generic hyperplane through (0) defined by $u_{1} X_{1}+\cdots+u_{n} X_{n}=0$. We shall use $H_{u}$ in two senses whenever it is proper: (1) $H_{u}$ means the linear polynomial $u_{1} X_{1}+\cdots+u_{n} X_{n}$ in $k(u)[X] \quad\left(=k\left(u_{1}, \cdots, u_{n}\right)\right.$ $\left.\left[X_{1}, \cdots, X_{n}\right]\right)$, (2) $H_{u}$ stands for the hyperplane defined by $u_{1} X_{1}+\cdots+$ $u_{n} X_{n}=0$. Let $k(u)=k\left(u_{1}, \cdots, u_{n}\right), V$ is a variety over $k(u)$ and $V \cap H_{u}$ is defined over $k(u)$. Let $\left(\mathfrak{p}, H_{u}\right)=\mathfrak{q}_{1} \cap \cdots \cap \mathfrak{q}_{t}$ be an irredundant primary decomposition with $\mathfrak{p}_{1}, \cdots, \mathfrak{p}_{t}$ as the associated prime ideals. Let $\mathfrak{p}_{1}, \cdots, \mathfrak{p}_{s}, s \leqq t$, be the isolated prime ideals. Since $(0) \in V$, $\left(\mathfrak{p}, H_{u}\right) \subset\left(X_{1}, \cdots, X_{n}\right) \cdot k(u)[X]$. Hence $\left(X_{1}, \cdots, X_{n}\right) \cdot k(u)[X]$ must contain at least one of the $\mathfrak{p}_{i}, i \leqq s$, say $\mathfrak{p}_{1}$. Let us denote $\mathfrak{p}_{1}$ by $\mathfrak{p}_{u}$ and let $W_{u}$ be the variety over $k(u)$ of $\mathfrak{p}_{u} \cdot W_{u}$ is of dimension $r-1$ as it is well known that any component of $V \cap H$, where $H$ is a hypersurface, is of dimension $r-1$. Let $(\xi)$ be a generic point of $W_{u}$ over $k(u)$. Since tr. $\operatorname{deg}_{k(\xi)} k(u ; \xi)+$ tr. $\operatorname{deg}_{k} k(\xi)=$ tr. $\operatorname{deg}_{k} k(u ; \xi)=$ tr. $\operatorname{deg}_{k}$ $k(u)+$ tr. $\operatorname{deg}_{k(u)} k(u ; \xi)=n+r-1$ and tr. $\operatorname{deg}_{k(\xi)} k(u ; \xi) \leqq n-1$, we have tr. $\operatorname{deg}_{k(\xi)} k(u ; \xi) \geqq r$. But $(\xi) \in V$, therefore tr. $\operatorname{deg}_{k} k(\xi)=r$. We thus have

Lemma 2. If $\operatorname{dim} V \geqq 2$, a generic point of $W_{u}$ over $k(u)$ is also a generic point of $V$ over $k$.

LEMMA 3. If $\xi_{j} \neq 0$, then $u_{1}, \cdots u_{j-1}, u_{j+1}, \cdots, u_{n}$ are algebraically independent over $k(\xi)$.

Proof. Say

$$
\begin{aligned}
i= & 1, \operatorname{tr} . \operatorname{deg}_{k\left(u_{2}, \cdots, u_{n}\right)} k\left(u_{1}, \cdots, u_{n} ; \xi\right) \\
& +\operatorname{tr} . \operatorname{deg}_{k} k\left(u_{2}, \cdots u_{n}\right)=n+r-1 .
\end{aligned}
$$

Therefore tr. $\operatorname{deg}_{k\left(u_{2}, \cdots, u_{n}\right)} k\left(u_{1}, \ldots, u_{n} ; \xi\right)=r$. Since

$$
\frac{u_{2} \xi_{2}+\cdots+u_{n} \xi_{n}}{\xi_{1}} \in k\left(u_{2}, \cdots, u_{n} ; \xi_{1}, \cdots, \xi_{n}\right),
$$

we have $k\left(u_{1}, \cdots, u_{n} ; \xi\right)=k\left(u_{2}, \cdots, u_{n} ; \xi\right)$. Now

$$
\text { tr. } \operatorname{deg}_{k(\zeta)} k\left(u_{2}, \cdots, u_{n} ; \zeta\right)+r=r+n-1 .
$$

Therefore $\operatorname{tr} . \operatorname{deg}_{k(\xi)} k\left(u_{2}, \cdots, u_{n} ; \xi\right)=n-1$, i.e., $\nu_{2}^{\prime}, \cdots, u_{n}$ are algebraically independent over $k(\xi)$.

Proposition 4. Let ( $(\xi), \mathfrak{p}_{u}$ and $W_{u}$ be as above. Then (p, $\left.H_{u}\right)$ : 
$\left(X_{1}, \cdots, X_{u}\right)^{\rho}=\mathfrak{p}_{u}$ for sufficiently large integers $\rho$, where $\left(X_{1}, \cdots, X_{n}\right)=$ $\left(X, \cdots, X_{n}\right) \cdot k(u)[X]$.

Proof. Let $F\left(u_{1}, \cdots, u_{n} ; X\right) \in \mathfrak{p}_{u}$ be a polynomial, we may assume $F\left(u_{1}, \cdots, u_{n} ; X\right) \in k\left[u_{1}, \cdots, u_{n}\right][X]$. If $\xi_{1} \neq 0, F\left(u_{1}, \cdots, u_{n} ; \xi\right)=0 \mathrm{im}-$ plies that $F\left(-\left(u_{2} \xi_{2}+\cdots+u_{n} \zeta_{n} / \xi_{1}\right), u_{2}, \cdots, u_{n} ; \xi\right)=0$. Hence there exists a nonnegative integer $\sigma$ such that $X_{1}^{\sigma}$.

$$
F\left(-\frac{u_{2} X_{2}+\cdots+u_{n} X_{n}}{X_{1}}, u_{2}, \cdots, u_{n} ; X\right) \in k\left(u_{2}, \cdots, u_{n}\right)[X]
$$

vanishes at $(\xi)$. By Lemma 3 , the prime ideal determined by $(\xi)$ in $k\left(u_{2}, \cdots, u_{n}\right)[X]$ is $\mathfrak{p} k\left(u_{2}, \cdots, u_{n}\right)[X]$. Thus

$$
X_{1}^{\sigma} F\left(-\frac{u_{2} X_{2}+\cdots+u_{n} X_{u}}{X_{1}}, u_{2}, \cdots, u_{n} ; X\right) \in \mathfrak{p} \cdot k\left(u_{1}, \cdots, u_{n}\right)[X]
$$

for sufficiently large $\sigma$. But

$$
\begin{aligned}
& X_{1}^{\sigma} F\left(-\frac{u_{2} X_{2}+\cdots+u_{n} X_{n}}{X_{1}}, u_{2}, \cdots, u_{n} ; X\right) \\
& -X_{1}^{\sigma} F\left(u_{1}, \cdots, u_{n} ; X\right) \equiv 0
\end{aligned}
$$

$\bmod \left(u_{1} X_{1}+\cdots+u_{n} X_{n}\right) \cdot k(u)[X]$ for sufficiently large $\sigma$. We have $X_{1}^{\sigma} F\left(u_{1}, \cdots, u_{n} ; X\right) \in\left(\mathfrak{p}, H_{u}\right) \cdot k(u)[X]$ for sufficiently large $\sigma$. The above discussion is symmetric with respect to those $\xi_{i} \neq 0$. Therefore for any $\xi_{i} \neq 0$, we have $X_{i}^{\sigma_{i}} F\left(u_{1}, \cdots, u_{n} ; X\right) \in\left(\mathfrak{p}, H_{u}\right)$ for sufficiently large integer $\sigma_{i}$ and for all $F \in \mathfrak{p}_{u}$. For any $j$ such that $\xi j=0, X_{j} \in \mathfrak{p}$. Thus $X_{j}^{\sigma_{j}} F \in\left(\mathfrak{p}, H_{u}\right)$ for any positive integer $\sigma_{j}$ and for all $F \in \mathfrak{p}_{u}$. Thus $\left(\mathfrak{p}, H_{u}\right):\left(X_{1}, \cdots, X_{n}\right)^{\rho} \supset \mathfrak{p}_{u}$ for sufficiently large integer $\rho$. We now show the other inclusion. Let $g\left(u_{1}, \cdots, u_{n} ; X\right)$ be an element in $\left(\mathfrak{p}, H_{u}\right):\left(X_{1}, \cdots, X_{n}\right)^{\rho}$. Then for any $h\left(u_{1}, \cdots, u_{n} ; X\right) \in\left(X_{1}, \cdots, X_{n}\right)^{\rho}$, $h(u ; X) \cdot g(u ; X) \in\left(\mathfrak{p}, H_{u}\right)$. Therefore, there exists $m_{i}(u ; X), n(u ; X) \in$ $k(u)[X]$ such that $h(u ; X) g(u ; X)=\sum_{s=1}^{s} m_{i}(u ; X) \cdot F_{i}(X)+n(u ; X) H_{u}$, where $\left(F_{1}, \cdots, F_{s}\right) \cdot k[X]=\mathfrak{p}$. Thus $h(u ; \xi) g(u ; \xi)=0$. If $g(u ; \xi) \neq 0$, then $h(u ; X)=0$ at $(\xi)$ for all $h(u ; X) \in\left(X_{1}, \cdots, X_{n}\right)^{\rho}$, which implies that $(\xi)=(0)$, a contradiction. Thus $g(u ; X)=0$ at $(\xi)$ and therefore $\mathfrak{p} \supset\left(p, H_{u}\right):\left(X_{1}, \cdots, X_{n}\right)^{\rho}$.

CoRollary. ( $\left(\mathfrak{p}, H_{u}\right)$ has only one isolated component.

Proof. Suppose $\mathfrak{p}_{2}$ is another isolated component, by Proposition 4, we have $\left(\mathfrak{p}, H_{u}\right):\left(X_{1}, \cdots, X_{n}\right)^{\rho^{\prime}}=\mathfrak{p}_{2}$, for sufficiently large integer $\rho^{\prime}$. Hence we have $\mathfrak{p}_{2}=\left(\mathfrak{p}, H_{u}\right)=\left(X_{1}, \cdots, X_{n}\right)^{\rho}=\mathfrak{p}_{u}$.

THEOREM 1. If $V / k$ is of dimension $r \geqq 2$, then $\left(\mathfrak{p}, H_{u}\right) \cdot k(u)[X]$ 
is either a prime ideal $\mathfrak{p}_{u}$ or an intersection of the prime ideal $\mathfrak{p}_{u}$ with a primary ideal of which $\left(X_{1}, \cdots, X_{n}\right) \cdot k(u)[X]$ is its radical.

Proof. Let $\mathfrak{B}=\left(\mathfrak{p}, H_{u}\right)$ and let $\mathfrak{B}=\mathfrak{q}_{1} \cap \cdots \cap \mathfrak{q}_{t}$ be the irredundant primary representation of $\mathfrak{B}$ with $\mathfrak{p}_{1}, \cdots, \mathfrak{p}_{t}$ as the associated prime ideals. By the corollary, there exists only one isolated prime component, say $\mathfrak{q}_{i}$, and denote $\mathfrak{p}_{1}$ by $\mathfrak{p}_{u}$. Let $\mathfrak{m}=\left(X_{1}, \cdots, X_{n}\right) \cdot k(u)[X]$. Since $\mathfrak{B}: \mathfrak{m}^{\rho}=\mathfrak{p}_{u}$ for sufficiently large $\rho$, we have $\left(\mathfrak{q}_{i}: \mathfrak{m}^{\rho}\right)=\mathfrak{p}_{u}$. There are two possibilities (I) no $\mathfrak{p}_{i}$ contains $\mathfrak{m}^{\lambda}$ for any nonnegative integer $\lambda$, or (II) some of $\mathfrak{p}_{i}$ contains a power of $\mathfrak{m}$. (I) leads to $\mathfrak{B}=\mathfrak{p}_{u}$. In case of (II), say $\mathfrak{p}_{2}$ contains $\mathfrak{m}^{\lambda}$ for some $\lambda$ then $\mathfrak{m}=\mathfrak{p}_{2}$. We may assume that there is no other $\mathfrak{p}_{j}$ to contain $\mathrm{m}^{\lambda}$ for any $0 \leqq \lambda \in \mathbf{Z}$. Thus for $i=1,3,4, \cdots r, \mathfrak{q}_{i}: \mathfrak{m}^{\lambda}=\mathfrak{q}_{i}$ for any $0 \leqq \lambda \in \mathbf{Z}$. Since $\mathfrak{q}_{2}: \mathfrak{m}^{\rho}=k(u)[X]$ for large $\rho$, hence $\mathfrak{B}: \mathfrak{m}^{\rho}=\left(\mathfrak{q}_{i}: \mathfrak{m}^{\rho}\right) \cap\left(\mathfrak{q}_{2}: \mathfrak{m}^{\rho}\right) \cap \cdots \cap\left(\mathfrak{q}_{\gamma}: \mathfrak{m}^{\rho}\right)=\mathfrak{q}_{1} \cap \mathfrak{q}_{3} \cap$ $\mathfrak{q}_{4} \cap \cdots \cap \mathfrak{q}_{t}$ and thus $\mathfrak{p}_{u} \cap \mathfrak{q}_{2}=\left(\mathfrak{p}, H_{u}\right)$.

CoRollary 1. If $V$ is normal over $k$, then $\left(\mathfrak{p}, H_{u}\right)=\mathfrak{p}_{u}$.

Proof. Passing to the coordinate ring of $V, k(u)[\eta]$, we have that $\left(u_{1} \eta_{1}+\cdots+u_{n} \eta_{n}\right) \cdot k(u)[\eta]$ is unmixed. Letting $\overline{\mathfrak{p}}_{u}=\mathfrak{p}_{u} / \mathfrak{p}, \overline{\mathfrak{q}}_{2}=\mathfrak{q}_{2} / \mathfrak{p}$ we have $\left(\sum u_{i} \eta_{i}\right)=\overline{\mathfrak{p}}_{u} \cap \overline{\mathfrak{q}}_{2}$ or $\left(\sum u_{i} \eta_{i}\right)=\overline{\mathfrak{p}}_{u}$, by Theorem 1. The unmixedness implies that $\left(\sum u_{i} \eta_{i}\right)=\overline{\mathfrak{p}}_{u}$, i.e., $\left(\mathfrak{p}, H_{u}\right)=\mathfrak{p}_{u}$.

COROLlary 2. If $V$ is k-normal at $(0)$, then $\left(\mathfrak{p}, H_{u}\right)=\mathfrak{p}_{u}$ i.e., $\left(\mathfrak{p}, H_{u}\right)$ is a prime ideal.

Proof. By Theorem 1, $\left(\mathfrak{p}, H_{u}\right)=\mathfrak{p}_{u}$ or $\left(\mathfrak{p}, H_{u}\right)=\mathfrak{p}_{u} \cap \mathfrak{q}_{2}$. Passing to the local ring $k(u)[\eta]_{(0)}$ of $V$ at $(0)$, we have $\left(\sum u_{i} \eta_{i}\right) k(u)[\eta]_{(0)}=\overline{\mathfrak{p}}_{u}^{e}$ or $\overline{\mathfrak{p}}_{u}^{e} \cap \overline{\mathfrak{q}}_{2}^{e}$ where $\overline{\mathfrak{p}}_{u}=\mathfrak{p}_{u} / \mathfrak{p}, \overline{\mathfrak{q}}_{2}=\mathfrak{q}_{2} / \mathfrak{p} \overline{\mathfrak{p}}_{u}^{e}$ and $\overline{\mathfrak{q}}_{2}^{e}$, are extensions of $\overline{\mathfrak{p}}_{u}$ and $\overline{\mathfrak{q}}_{2}$ in $k(u)[\eta]_{(0)}$ respectively. Since $k(u)[\eta]_{(0)}$ is integrally closed, the unmixedness of $\left(\sum u_{i} \eta_{i}\right) \cdot k(u)[\eta]_{(0)}$ implies that $\left(\sum u_{i} \eta_{i}\right) k(u)[\eta]=\bar{p}_{u}$ and $\left(\mathfrak{p}, H_{u}\right)=\mathfrak{p}_{u}$.

Recall that $V / k$ is a quasi-absolutely irreducible variety if $k$ is quasi-algebraically closed in the field $k\left(\xi_{1}, \cdots, \xi_{n}\right)$ of rational functions on $V / k$; a prime ideal $\mathfrak{U}$ in $k\left[X_{1}, \cdots, X_{n}\right]$ is quasi-absolutely irreducible if $\bar{k}\left[X_{1}, \cdots, X_{n}\right] \mathfrak{X}$ is primary, where $\bar{k}$ is the algebraic closure of $k$. By [11; Th. 10, p. 371], $\mathfrak{p}$ is quasi-absolutely irreducible if and only if $V / k$ is quasi-absolutely irreducible. $V / k$ is absolutely irreducible if $k$ is algebraically closed in $k(\xi)$ and $k(\xi)$ is separable over $k$. A prime ideal $\mathfrak{i}$ in $k\left[X_{1}, \cdots, X_{n}\right]$ is absolutely irreducible if $\bar{k}\left[X_{1}, \cdots, X_{n}\right] . \quad \mathfrak{A}$ is a prime ideal. It is well known that the prime ideal $\mathfrak{p}$ of $V / k$ is absolutely irreducible if and only if $V / k$ is.

THEOREM 2. If $V / k$ is quasi-absolutely irreducible of dimension 
$r \geqq 3$ and if $k$ is infinite, then $V \cap H_{u} / k(u)$ is quasi-absolutely irreducible.

Proof. Let $(\eta)$ be a generic point of $V \cap H_{u}$ over

$$
k(u)=k\left(u_{1}, \cdots, u_{n}\right) .
$$

By Lemma 2, $(\eta)$ is a generic point of $V$ over $k$. Let $\eta_{1}, \eta_{2}$, and $\eta_{n}$ be algebraically independent over $k$. By Lemma 3, $(\eta)$ is a generic point of $V$ over $k\left(u_{2}, \cdots, u_{n}\right)$. By [11; Lemma 5, p. 368], $k\left(u_{2}, \cdots, u_{n}\right)$ is quasi-algebraically closed in $k\left(u_{2}, \cdots, u_{n}\right)(\eta)$. Let $\Sigma=k\left(u_{2}, \cdots, u_{n-1}\right)$ $(\eta), u_{n}$ is algebraically independent over $\Sigma$. Viewing $k\left(u_{2}, \cdots, u_{n-1}\right)$ as the field $k$ and $u_{n}$ as the $u$ in [11; corollary, p. 369], we have $\Sigma\left(u_{n}\right)=$ $k\left(u_{2}, \cdots, u_{n-1}\right)\left(u_{n}\right)(\eta)=k(u)(\xi)$. Let $\xi_{1}$ and $\xi_{2}$ in [11; corollary, p. 369] be replaced by $-\left(u_{2} \eta_{2}+\cdots+u_{n-1} \eta_{n-1}\right) / \eta_{1}$ and $-\eta_{n / n_{1}}$ respectively, one sees that $-\left(u_{2} \eta_{2}+\cdots+u_{n-1} \eta_{n-1}\right) / \eta_{1}$ and $\eta_{n} / \eta_{1}$ are algebraically independent over $k\left(u_{2}, \cdots, u_{n-1}\right)$. Hence by the same corollary we have that

$$
\begin{aligned}
& k\left(u_{2}, \cdots, u_{n-1}\right)\left(u_{n}\right)\left(-\left(u_{2} \eta_{2}+\cdots+u_{n-1}\right) / \eta_{1}-u_{n} \eta_{n} / \eta_{1}\right) \\
= & k\left(u_{2}, \cdots, u_{n-1}\right)\left(u_{n}\right)\left(u_{1}\right)=k(u)
\end{aligned}
$$

quasi-algebraically closed in $\Sigma\left(u_{n}\right)=k(u)(\eta)$.

LEMMA 4. Let $K$ be a regular finitely generated extension of an infinite field $k$ with tr. $\operatorname{deg}_{k} K \geqq 3$. Let $x, y, z$ be three elements of $K$ algebraically independent over $k$, and $z / x \notin K^{p} k$, where $p$ is the characteristic of $k$. Then for all but a finite number of constants $c \in k, K$ is a regular extension of $k(y+c z / x)$. Moreover, let $\tau$ be an indeterminate $K(\tau)$ is regular over $k(\tau)(y+\tau z / x)$.

\section{Proof. [5; Lemma 3].}

THEOREM 3. If $V / k$ is an absolutely irreducible variety of dimension $r \geqq 3$ defined over an infinite field $k$, then $V \cap H_{u} / k(u)$ is an absolutely irreducible variety.

Proof. $V \cap H_{u} / k(u)$ is irreducible. Let $(\xi)$ be a generic point of $V \cap H_{u}$ over $k(u)$. By Lemma 3 , $(\xi)$ is a generic point of $V$ over $k$, hence $\operatorname{tr} . \operatorname{deg}_{k} k(\xi) \geqq 3$ and $k(\xi)$ is a regular extension over $k$ by [12; Proposition 1, p. 69]. Let $\xi_{1}, \xi_{2}$ and $\xi_{n}$ be three elements in a separable transendental basis of $k(\xi)$ over $k$. Let $K=k\left(u_{2}, \cdots, u_{n-1}\right)(\xi), u_{n}$ is algebraically independent over $K$. Viewing $k\left(u_{2}, \cdots, u_{n-1}\right)$ as the field $k$ and $u_{n}$ as the $\tau$ in Lemma 4 , we have $K\left(u_{n}\right)=k(u)(\xi)$. Let $y=-\left(u_{2} \xi_{2}+\cdots+u_{n-1} \xi_{n-1}\right), z=\xi_{n}$ and $x=\xi_{1}$, then $x, y$ and $z$ are 
algebraically over $k\left(u_{2}, \cdots, u_{n-1}\right)$. By [6, Proposition 1, p. 185] and [6; corollary to Proposition 2, p. 186], $z / x=-\xi_{n} / \xi_{1} \notin K^{p} k\left(u_{2}, \cdots, u_{n-1}\right)$, we have that $K\left(u_{n}\right)$ is a regular extension over

$$
k\left(u_{v_{2}}, \cdots, u_{n-1}\right)\left(u_{n}\right)\left(\frac{y-u_{n} z}{k}\right)=k(u) .
$$

Therefore $k(u)(\xi)$ is a regular extension over $k(u)$, hence $V \cap H_{n} / k(u)$ is an absolutely irreducible variety.

Let $\left\{F_{1}, \cdots, F_{s}\right\}$ be a set of generators of $\mathfrak{p}$ in $k[x]$. Let $P$ be a point on $V$. According to [14], $P$ is $k$-simple on $V$ if and only if the mixed Jacobian of $\left\{F_{1}, \cdots, F_{s}\right\}$ is of rank $n-r$ at $P$. When $k(P)$ is separable over $k, P$ is $k$-simple on $V$ if and only if the classical Jacobian of $\left\{F_{1}, \cdots, F_{s}\right\}$ is of rank $n-r$ at $P$.

Following Theorem 1, we denote $\mathfrak{p}_{u}$ as the sole isolated component of $\left(\mathfrak{p}, H_{u}\right)$ and $W_{u} / k(u)$ as its variety in the sequel.

THEOREM 4. Let $V / k$ be of dimension $r \geqq 2$. Then $P \in W_{u}$ is $k(u)$-simple if and only if $P$ is k-simple on $V$.

Proof. Let $P \in W_{u}$ be $k$-simple on $V$. By Theorem 1, $\left(\mathfrak{p}, H_{u}\right)=$ $\mathfrak{p}_{u} \cap \mathfrak{A}$, where $\mathfrak{Y}$ is the embedded component with $\left(X_{1}, \cdots, X_{n}\right)$ as radical. Let $(\eta)$ be a generic point of $V$ over $k(u)$, and let $(\xi)$ be a generic point of $W_{u}$ over $k(u)$. Let $k(u)[\eta]_{p}$ and $k(u)[\xi]_{p}$ be the local rings of $V$ and $W_{u}$ at $P$ respectively. $k(u)[\eta]_{p}$ is regular and

$$
k(u)[\xi]_{p} \cong k(u)[\eta]_{p} / \bar{p}_{u} \cdot k(u)[\eta]_{p},
$$

where $\overline{\mathfrak{p}}_{u}$ is the residue of $\mathfrak{p}_{u}$ modulo $\mathfrak{p}$. If $P \neq(0)^{1}$, let $\mathfrak{X}$ be the residue of $\mathfrak{X}$ modulo $\mathfrak{p}$ and let $\mathfrak{m}_{p}$ be the maximal ideal of $k(u)[\eta]_{p}$, then $\mathfrak{A k}(u)[\eta] \not \subset \mathfrak{m}_{p}$. For otherwise $\left(\eta_{1}, \cdots, \eta_{n}\right)^{\rho} \subset \mathfrak{m}_{p}$ for some integer $\rho>0$, as $\left(X_{1}, \cdots, X_{n}\right)^{\rho} \subset \mathfrak{A}$. Thus $P=(0)$, a contradiction. Therefore, when $P \neq(0),\left(\Sigma u_{i} \eta_{i}\right) \cdot k(u)[\eta]_{p}=\overline{\mathfrak{p}}_{u} \cdot k(u)[\eta]_{p}$, and $k(u)[\xi]_{p} \cong k(u)[\eta]_{p} /$ $\left(\Sigma u_{i} \eta_{i}\right) k(u)[\eta]_{p}$. By [16; Th. 26, p. 303], to show that $k(u)[\xi]_{p}$ is regular it is sufficient to show that $\sum u_{i} \eta_{i} \notin \mathfrak{m}_{p}^{2}$. But this is the case, for if $\sum u_{i} \eta_{i} \in \mathfrak{M}_{p}^{2}$, taking partial derivatives with respect to $u_{i}$ for $i=$ $1,2, \cdots, n$, we have $\eta_{i} \in \mathfrak{m}_{p}$ for $i=1,2, \cdots, n$, i.e., $P=(0)$ a contradiction. Therefore $k(u)[\xi]_{p}$ is regular. If $P=(0)$, then $(0)$ is $k$ normal on $V$. By Corollary 2 to Theorem 1 , $\left(\mathfrak{p}, H_{u}\right)=\mathfrak{p}_{u}$. In viewing [14, Th. 7, p. 28], we let $F_{1}, \cdots, F_{s}$ be a basis of $\mathfrak{p}$, and let $F_{i}$ 's and $X_{i}$ 's be so arranged that $\left(\operatorname{det}\left(\partial F_{i} / \partial X_{j}\right)\right)_{(0)} \neq 0$, where $i, j=1,2, \cdots$, $n-r$, and the subscript $(0)$ means that we replace $(X)$ by $(0)$ after the determinant of the Jacobian is formed, as the rank of

${ }^{1}$ If $P \neq 0$, and if $P$ is $k$-simple on $V$, then $P$ remains simple on $W_{u} / k(u)$ follows also from [13; the theorem of Bertini, p. 138]. 


$$
J\left(F_{1}, \cdots, F_{s}, X_{1}, \cdots, X_{n}\right)_{(0)}=n-r .
$$

Consider

$$
\Delta_{\jmath}=\operatorname{det}\left[\begin{array}{cccc}
\partial F_{1} / \partial X_{1} & \cdots & \partial F_{1} / \partial X_{n-r} & \partial F_{1} / \partial X_{j} \\
\vdots & & & \\
\partial F_{n-r} / \partial X_{1} & \cdots & \partial F_{n-r} / \partial X_{n-r} & \partial F_{n-r} / \partial X_{j} \\
u_{1} & \cdots & u_{n-r} & u_{j}
\end{array}\right]_{(0)}
$$

where $\eta-r+1 \leqq j<\eta$. If $\Delta_{j}=0$ for some $j$ then $u_{1}, \cdots, u_{n-r}, u_{j}$ are algebraically dependent over $k$. This is a contradiction, hence (0) is $k$-simple on $W_{u}$. Conversely, assume that $P \in W_{u}$ is $k(\nu j)$-simple on $W_{u}$. If $P \neq(0)$, we have $k(u)[\xi]_{p} \cong k(u)[\eta]_{p} /\left(\Sigma u_{i} \eta_{2}\right) \cdot k(u)[\eta]_{p}$ from the above. If $P=(0)$, then $P$ is $k(u)$-normal on $W_{u}$. By Theorem 6 in the following $V / k$ is normal at $(0)$, therefore $\left(\mathfrak{p}, H_{u}\right)=\mathfrak{p}_{u}$ and $k(u)[\xi]_{(0)} \cong$ $k(u)[\eta]_{(0)} /\left(\Sigma u_{i} \eta_{i}\right) \cdot k(u)[\eta]_{(0)}$. Therefore $k(u)[\xi]_{p} \cong k(u)[\eta]_{p} /\left(\Sigma u_{i} \eta_{i}\right) \cdot k(u)[\eta]_{p}$ if $P$ is $k(u)$-simple on $W_{u}$. Since $h t\left(\left(\Sigma u_{i} \eta_{i}\right) \cdot k(u)[\eta]_{p}\right)=1$, it follows from $\left[8 ;(9 ; 11)\right.$, p. 28] that $k(u)[\eta]_{p}$ is a regular local ring. Hence $P$ is $k$-simple on $V$.

By an argument similar to the proof of Lemma 2, we have the following.

Corollary. If $V / k$ is of dimension $r \geqq 3$ and if $V / k$ is locally free of $(r-1)$-dimensional singularities, then $V \cap H_{u} / k(u)$ is locally free of $(r-2)$-dimensional singularities.

Note. If $r=2$, the corollary is clearly false as one sees by taking $V$ to be a cone with vertex at $(0)$.

THEOREM 5. If $V / k$ is a complete intersection of dimension $\geqq 3$ and if $V$ is k-normal at $(0)$, then the generic hyperplane section $V \cap H_{u}$ is also $k(u)$-normal at (0).

Proof. $V / k(u)$ is $k(u)$-normal at $(0)$, by Lemma 1 . By corollary to Theorem 1, $\left(\mathfrak{p}, H_{u}\right)=\mathfrak{p}_{u}$ is prime. For any polynomial $F \neq 0$ in $k(u)[X]$, by [7; Th. p. 49] or [16; Th. 26, p. 203], $\left(\mathfrak{p}_{u}, F\right)=\left(\mathfrak{p}, H_{u}, F\right)$ is unmixed. Hence, passing to the quotient modulo $\mathfrak{p}_{u}$, we have that every nonzero principal ideal in the coordinate ring $k(u)[\xi]$ of $V \cap H_{u}$ is unmixed. It follows that every nonzero principal ideal in the local ring of $V \cap H_{u}$ at $(0), k\left(v_{0}\right)[\xi]_{(0)}$, is also unmixed. Since $V / k$ is $k$-normal at $(0)$, therefore $V / k$ is locally free of $(r-1)$-dimensional singularities at (0). By the above corollary, $V \cap H_{u}$ is locally free of $(r-2)$-dimensional singularities at (0). It follows from Proposition 1 that $V \cap H_{u}$ is $k(u)$-normal at $(0)$. 
THEOREM 6. If $V \cap H_{u}$ is $k(u)$-normal at $(0)$, then $V / k$ is normal at $(0)$.

Proof. This theorem is really a consequence of [3; Lemma 4, p. 360] ([8; (36.9), p. 134]). Indeed, let $(\eta)$ be a generic point of $V$ over $k(u)$. Passing to $k(u)[\eta]$, by Theorem 1 , we have $\left(u_{1} \eta_{1}+\cdots+u_{n} \eta_{n}\right) \cdot$ $k(u)[\eta]=\overline{\mathfrak{p}}_{u} \cap \overline{\mathfrak{q}}$, where $\overline{\mathfrak{p}}_{u}$ and $\overline{\mathfrak{q}}$ are residues of $\mathfrak{p}_{u}$ and $\mathfrak{q}$ modulo $\mathfrak{p}$ respectively. It is clear that (1) $\left(u_{1} \eta_{1}+\cdots+u_{n} \eta_{n}\right) \cdot k(u)[\eta]_{(0)}=\overline{\mathfrak{p}}_{u} \cdot$ $k(u)[\eta]_{(0)} \cap \overline{\mathrm{q}} k(u)[\eta]_{(0)}, u_{1} \eta_{1}+\cdots+u_{n} \eta_{n}$ is in the Jacobson radical of $k(u)[\eta]_{(0)}, \quad(2) \quad\left(u_{1} \eta_{1}+\cdots+u_{n} \eta_{n}\right) \cdot\left(k(u)[\eta]_{(0)}\right)_{\bar{p}_{u}}=\bar{p}_{u} \cdot\left(k(u)[\eta]_{(0)}\right)_{\bar{p}_{u}}$, and (3) let $(\xi)$ be a generic point of $V \cap H_{u}$ over $k(u)$, then

$$
\frac{k(u)[\eta]_{(0)}}{\overline{\mathrm{p}}_{u} k(u)[\eta]_{(0)}} \cong k(u)[\xi]_{(0)},
$$

which is integrally closed as $V \cap H_{u}$ is $k(u)$-normal at (0). Moreover, let $k(u)[\eta]_{(0)}^{*}$ be the integral closure of $k(u)[\eta]_{(0)}$ in $k(u)(\eta)$, and let $\mathfrak{p}^{\prime}$ be a minimal prime divisor of $\left(u_{1} \eta_{1}+\cdots+u_{n} \eta_{n}\right) \cdot k(u)[\eta]_{(0)}^{*}$. It follows from [2; Th. 2, p. 253] and [2; Th. 3; p. 254] that $h t\left(\mathfrak{p}^{\prime} \cap k(u)[\eta]_{(0)}\right)=$ $h t \mathfrak{p}=1$. Therefore $\mathfrak{p}^{\prime} \cap k(u)[\eta]_{(0)}=\overline{\mathfrak{p}}_{u}$, i.e., every minimal prime divisor of $\left(u_{1} \eta_{1}+\cdots+u_{n} \eta_{n}\right) \cdot k(u)[\eta)_{(0)}^{*}$ lies over $\mathfrak{p}_{u}$. The above verify the conditions of [3; Lemma 4, p. 360], therefore $k(u)[\eta]_{(0)}$ is integrally closed.

3. The local normal problem. Throughout this section let $V / k$ be a variety of dimension $r \geqq 3$, passing through (0) with $(\xi)$ as a generic point over $k$ and let $H_{u}: u_{1} X_{1}+\cdots+u_{n} X_{n}=0$ be a generic hyperplane through (0). If $V / k$ is normal at (0), is it true that $H_{u} \cap V$ $k(u)$-normal at (0)? If $V / k$ is a complete intersection then by Theorem 5 , the answer to the question is yes. However we shall prove the answer to the question is negative in general.

DEFINITION 4. (a) Let $R$ be a Noetherian ring. Subset $\left\{a_{1}, \cdots, a_{q}\right\}$ of $R$ is a prime sequence if for each $i=1,2, \cdots, q, a_{i}$ is not a zero divisor in the ring $R /\left(a_{1}, \cdots, a_{i-1}\right) \cdot R$.

(b) Let $R$ be a local ring, the number of elements of a maximal prime sequence in $R$ is called the homological co-dimension of $R$, and is denoted by $\operatorname{cod} h(A)$. If $\operatorname{cod} h(A)=\operatorname{dim} A$, we say that $A$ is a CohenMacaulay ring.

For a general commutative ring $R$ and a multiplicative system $S$ which does not contain 0 , it is well known [15, p. 219] that (谁: $\mathfrak{B})^{e} \subset$ $\mathfrak{X} \mathfrak{C}^{e}: \mathfrak{B}^{e}$ and $\left.(\mathfrak{X}: \mathfrak{Y})^{c} \subset \mathfrak{X}^{c}: \mathfrak{Y}\right)^{c}$, where $\left({ }^{*}\right)^{e}=\left(^{*}\right) \cdot R_{S},\left(^{*}\right)^{c}=f^{-1}\left({ }^{*}\right), f$ is the canonical homomorphism of $R$ into $R_{S}$ and where $\mathfrak{Y}, \mathfrak{B}$ are two ideals in $R$, and $\mathfrak{X}, \mathfrak{Y}$ are two ideals in $R_{s}$. 
Proposition 5. Let $\mathfrak{A}, \mathfrak{B}, \mathfrak{X}$ and $\mathfrak{Y}$ be the same as above. Then (a) $(\mathfrak{U}: \mathfrak{B})^{e}=\mathfrak{U}^{e}: \mathfrak{B}^{e}$; if $\mathfrak{A} \supset \operatorname{Ker} f$ and $\mathfrak{B}$ is finitely generated, also (b) $(\mathfrak{X}: \mathfrak{Y})^{c}=\mathfrak{X}^{c}: \mathfrak{Y}^{c}$ if $\mathfrak{Y}$ is finitely generated.

Proof. Let $\mathfrak{B}=\left(b_{1}, \cdots, b_{t}\right) R$, we have $\mathfrak{B}^{e}=\left(f\left(b_{1}\right), \cdots, f\left(b_{t}\right)\right) \cdot R_{s}$. Let $x \in \mathfrak{Y} \mathfrak{U}^{e}: \mathfrak{B}^{e}$. Then $x \mathfrak{B}^{e} \subset \mathfrak{U} \mathfrak{U}^{e}$ and $x f\left(b_{i}\right)=f\left(a_{i}\right) / f\left(s_{i}\right)$ for some $a_{i} \in \mathfrak{N}$ and $s_{i} \in S$. Therefore $f\left(\pi_{i} s_{i}\right) x f\left(b_{i}\right) \in f(\mathfrak{Q})$. For each $b \in f(\mathfrak{B}), b=\sum_{j} f\left(r_{j}\right) f\left(b_{j}\right)$ for some $r_{j} \in R$. Now $f\left(\pi_{i} s_{i}\right) x b=\sum_{j} f\left(\pi_{i} s_{i}\right) x f\left(r_{j}\right) f\left(b_{j}\right) \in f($ 泣 $)$, which implies that $f\left(\pi_{i} s_{i}\right) x \in f(\mathfrak{Y}): f(\mathfrak{B})$. Hence $x \in(f(\mathfrak{V}): f(\mathfrak{B})) R_{s}$. Since $\mathfrak{Y} \supset \operatorname{Ker} f$, by [15; (15), p. 148], $f(\mathfrak{I}): f(\mathfrak{B})=f(\mathfrak{Q Y}: \mathfrak{B})$. Therefore $x \in(\mathfrak{Y}: \mathfrak{B})^{e}$ and $\mathfrak{U} \mathfrak{H}^{e}: \mathfrak{B}^{e}=(\mathfrak{U}: \mathfrak{B})^{e}$. The proof of $(\mathrm{b})$ is similar.

Lemma 5. $k(u)[\xi]_{(0)}$ is Cohen-Macaulay if and only if $k[\xi]_{(0)}$ is Cohen-Macaulay, where $k[\xi]$ is the coordinate ring of $V / k$, and $u$ is an indeterminate over $k(\xi)$.

Proof. If $k[\xi]_{(0)}$ is Cohen-Macaulay, then there exist $\ell_{1}, \cdots \ell_{r}$ such that $\left\{\ell_{1}, \cdots \ell_{r}\right\}$ forms a maximal prime sequence, where $r=\operatorname{dim} V$. Thus $\left(\ell_{1}, \cdots, \ell_{i}\right) k[\xi]_{(0)}:\left(\ell_{i+1}\right) \cdot k[\xi]_{(0)}=\left(\iota_{1}, \cdots, \ell_{i}\right) \cdot k[\xi]_{(0)}$ for $i=1,2, \cdots r$. By [15; (1), p. 227], [15; (15), (21), p. 148] Proposition 5 and [16; (3), p. 221] one has $\left(\ell_{1} \cdots, \ell_{i}\right) k(u)[\xi]_{(0)}:\left(\ell_{i+1}\right) k(u)[\xi]_{(0)}=\left(\ell_{1}, \cdots, \ell_{i}\right) k(u)[\xi]_{(0)}$, for $i=1,2, \cdots, r$. Therefore $\left\{\ell_{1}, \cdots, \ell_{r}\right\}$ remains as a maximal prime sequence of $k(u)[\xi]_{(0)}$. Thus $k(u)[\xi]_{(0)}$ is Cohen-Macaulay.

Conversely, let $k(u)[\xi]_{(0)}$ be Cohen-Macaulay, let $\left\{\iota_{1}(u ; \xi), \cdots \iota_{r}(u ; \xi)\right\}$ be a maximal prime sequence of $k(u)[\xi]_{(0)}$. Then, for $i=1,2, \cdots, r$, we have $\left(\ell_{1}(u ; \xi), \cdots, \ell_{i}(u ; \xi)\right) \cdot k(u)[\xi]_{(0)}:\left(\ell_{i+1}(u ; \xi)\right) \cdot k(u)[\xi]_{(0)}=\left(\ell_{1}(u ; \xi), \cdots\right.$, $\left.\ell_{i}(u ; \xi)\right) \cdot k(u)[\xi]_{(0)}$. By [15; (21), p. 148], going back to the polynomial ring $k(u)[x]$, we have $\left(\ell_{1}(u ; x), \cdots, \ell_{i}(u ; x), \mathfrak{p}\right) k(u)[x]_{(0)}:\left(\ell_{i+1}(u ; x), \mathfrak{p}\right) k(u)[x]_{(0)}=$ $\left(\ell_{1}(u ; x), \cdots, \ell_{i}(u ; x), \mathfrak{p}\right) k(u)[x]_{(0)}$. In viewing [4; Satz 3, p. 59], one sees that

$$
\begin{aligned}
\overline{\left(\ell_{1}(u ; x), \cdots, \ell_{i}(u ; x), \mathfrak{p}\right) k(u)[x]_{(0)}:} & \overline{\left(\ell_{i+1}(u ; x), \mathfrak{p}\right) k(u)[x]_{(0)}}=\overline{\left(\ell_{1}(u ; x), \cdots, \ell_{i}(u ; x), \mathfrak{p}\right) k(u)[x]_{(0)}}
\end{aligned}
$$

almost always for $i=1,2, \cdots r$, where the bar means specialization of $u$ to elements in $k$. Passing to the local ring of $V / k(u)$ at $(0)$, by [15; (15), p. 148], we have $\overline{\ell_{1}\left(u ; \xi, \cdots, \ell_{i}(u ; \xi)\right) k(u)[\xi]_{(0)}: \ell_{i+1}(u ; \xi) k(u)[\xi]_{(0)}}=$ $\overline{\left(\ell_{1}(u ; \xi), \cdots, \ell_{i}(u ; \xi)\right) k(u)[\xi]_{(0)}}$ almost always for $i=1,2, \cdots r$. Let $a \in k$ be such that the above holds and $\ell_{i}(a ; \xi) \neq 0$, for $i=1,2, \cdots, r$, then $\left(\ell_{1}(a ; \xi), \cdots, \ell_{i}(a ; \xi)\right) k[\xi]_{(0)}:\left(\ell_{i+1}(a ; \xi)\right) k[\xi]_{(0)}=\left(\iota_{1}(a ; \xi), \cdots, \ell_{i}(a ; \xi)\right) \cdot k[\xi]_{(0)}$ for $i=1,2, \cdots r$. Therefore $\left\{\ell_{1}(a, \xi), \cdots, \ell_{r}(a, \xi)\right\}$ forms a system of prime sequence of $k[\xi]_{(0)}$. Hence $k[\xi]_{(0)}$ is Cohen-Macaulay.

THEOREM 7. Let $V / k$ and $H_{u}$ be the same as the above. It is not 
true in general that if $V / k$ is k-normal at (0), then $V \cap H_{u} / k(u)$ is $k(u)$-normal at $(0)$.

Proof. Suppose that if $V / k$ is $k$-normal at (0), then $V \cap H_{u} / k(u)$ is $k(u)$-normal at $(0)$. Let $(\xi)$ be a generic point of $V$ over $k$ and let $(\eta)$ be that of $V \cap H_{u}$ over $k(u)$. Applying the supposition to $V \cap H_{u} / k(u)$, we get $\left(V \cap H_{u}\right) \cap H_{u(2)} k(u, u(2))$-normal at $(0)$, where

$$
H_{u(2)}: u_{21} X_{1}+\cdots+u_{2 n} X_{n}=0
$$

is a generic hyperplane through $(0)$ on

$$
V \cap H_{u} / k(u) \text { and } u(2)=\left\{u_{21}, \cdots, u_{2 n}\right\}
$$

are algebraically independent over $k(u)(\xi, \eta)$. Repeating the supposition and Corollary 2 to Theorem 1 in this way until dimension $r$ of $V$ is cut down to 2 , we have then

$$
V \cap H_{u} \cap H_{u(2)} \cap \cdots \cap H_{u(r-2)} k(u, u(2), \cdots, u(\gamma-2)) \text {-normal }
$$

at $(0)$, where $u(i)=\left\{u_{i 1}, \cdots, u_{i n}\right\}$, and $\left\{u_{i 1}, \cdots, u_{i n}\right\}$ are indeterminates over $k\left(u, u(2), \cdots, u(i-1)\left(\xi, \eta, \eta_{2}, \eta_{i-1}\right)\right.$ with $\eta_{j}=\left(\eta_{j 1}, \cdots, \eta_{j n}\right)$ being a generic point of $V \cap H_{u} \cap H_{u(2)} \cap \cdots \cap H_{u(j)}$ over $k(u, u(2), \cdots u(j))$. Let $U=\{u, u(2), \cdots, u(\gamma-2)\}$, then $k(U)=k(u, u(2), \cdots, u(\gamma-2))$. Consider $V / k(U),(\xi)$ is a generic point of $V$ over $k(U)$. Correspondingly in the coordinate ring $k(U)[\xi]$ of $V$ over $k(U)$ we have then $r-2$ quantities $\ell_{i}=u_{i 1} \xi_{1}+\cdots+u_{i n} \xi_{n}, i=1,2, \cdots r-2$, such that $\left(\ell_{1}, \cdots, \ell_{i}\right)$ is a prime ideal in $k(U)[\xi]_{(0)}$ and $\ell_{i+1} \notin\left(\ell_{1}, \cdots, \ell_{i}\right) k(U)[\xi]_{(0)}$. Thus $\left\{\ell_{1}, \cdots, \ell_{r-2}\right\}$ is a prime sequence in the local ring $k(U)[\xi]_{(0)}$. Let $R$ be $k(U)[\xi]_{(0)} /\left(\ell_{1}, \cdots, \ell_{r-2}\right) \cdot k(U)[\xi]_{(0)}$, then $R$ is integrally closed of dimension 2. By [16; (3), p. 397], $R$ is Cohen-Macaulay. Let $a, b \in k(U)[\xi]_{(0)}$ be such that their residues modulo $\left(\ell_{1}, \cdots, \ell_{r-2}\right) \cdot k(U)[\xi]_{(0)}$ form a maximal prime sequence of $R$, then $\left\{\ell_{1}, \cdots, \ell_{r-2}, a, b\right\}$ is a prime sequence of $k(U)[\xi]_{(0)}$. Therefore $\operatorname{dim} k(U)[\xi]_{(0)}=\operatorname{cod} h k(U)[\xi]_{(0)}$ and hence $k(U)[\xi]_{(0)}$, is a Cohen-Macaulay ring. It follows from Lemma 5 that $k[\xi]_{(0)}$ is a Cohen-Macaulay ring. So under the supposition, we conclude that $k[\xi]_{(0)}$ is integrally closed implies that $k[\xi]_{(0)}$ is Cohen-Macaulay. But on the other hand, [1; Proposition, p. 655] and [1; Th. 5, p. 653] yield an example of a local ring of an algebraic variety at a rational point which is a factorial local ring (hence normal), but not a CohenMacaulay local ring. Hence the above supposition yields a contradiction.

THEOREM 8. If $V / k$ is normal at (0), and the local ring $k[\xi]_{(0)}$ is a Cohen-Macaulay ring, then $V \cap H_{u} / k(u)$ is normal at (0).

Proof. By the corollary to Theorem $4,\left(\mathfrak{p}, H_{u}\right)$ is free of $(\gamma-2)$ - 
dimensional singularities. By Lemma $5, k(u)[\xi]_{(0)}$ is Cohen-Macaulay. For any nonzero $a(u ; \xi)$ in $k(u)[\xi]_{(0)}$ not in the prime ideal

$$
\left(u_{1} \xi_{1}+\cdots+u_{n} \xi_{n}\right) \cdot k(u)[\xi]_{(0)},\left\{a(u, \xi), u_{1} \xi_{1}+\cdots+u_{n} \xi_{n}\right\}
$$

forms a prime sequence of $k(u)[\xi]_{(0)}$, therefore by [16; Lemma $5, \mathrm{p}$. 401], $\left(a(u, \xi), u_{1} \xi_{1}+\cdots+u_{n} \xi_{n}\right) \cdot k(u)[\xi]_{(0)}$, is unmixed. Hence every nonzero principal ideal of $k(u)[\xi]_{(0)} /\left(u_{1} \xi_{1}+\cdots+u_{n} \xi_{n}\right) \cdot k(u)[\xi]_{(0)}$, is unmixed. It follows from Proposition 1 that $V \cap H_{u}$ is $k(u)$-normal at (0).

I would like to take this opportunity to express my thanks to Professor A. Seidenberg for suggesting the problem, his valuable advice and continuous encouragement.

\section{BIBLIOGRAPHY}

1. M. J. Bertin, Anneaux d'invariants d'anneaux de polynomes, en characteristigne $p$, C. A. Acad. Sci. Paris, 264 (1967), 653-656.

2. I. S. Cohen and A. Seidenberg, Prime ideals and integral dependence, Bull. Amer. Math. Soc. 52 (1946), 252-261.

3. H. Hironaka, $A$ note on algebraic geometry over ground rings. The invarience of Hilbert characteristic function under the specialization process, Illinois J. Math. 2 (1958), 355-366.

4. W. Krull, Parameterspezialisierung in poloynomringen, Arch. Math. 1 (1948), 56-64.

5. W. Kuan, On the hyperplane sections through two given points of an algebraic variety, Canad. J. Math, 22 (1970), 128-133.

6. S. Lang, Introduction on Algebraic Geometry, Interscience, New York, 1964.

7. F. S. Macaulay, Algebraic Theory of Modular Systems, Cambridge Tracts Math., 19, Cambridge University Press, Cambridge, 1916.

8. M. Nagata, Local Rings, Interscience, New York, (1962).

9. E. Noether, Eliminations theorie und allgemeine Idealtheorie, Math. Ann. 90 (1923), 229-261.

10. P. Samuel, Methodes D'algebre abstraite en Geometrie Algebrique, Springer-verlag, Berlin 1967.

11. A. Seidenberg, The hyperplane section of normal varieties, Trans. Amer. Math. Soc. 69 (1950), 357-386.

12. A. Weil, Foundations of Algebraic Geometry, Amer. Math. Soc. Colloquium Publications, Vol. 241962.

13. O. Zariski, The theorem of Bertini on the variable singular points of a linear system of varieties, Trans. Amer. Math. Soc. 56 (1944), 130-140.

14. The concept of a simple point of an abstract algebraic variety, Trans. Amer. Math. Soc. 62 (1947), 1-52.

15. O. Zariski and P. Samuel, Commutative Algebra I, Van Nostrand, New York, 1958. 16. - - Samuel, Commutative algebra II, Van Nostrand, New York, 1960.

Received December 16. 1969. Part of the results of this paper over a field $k$ of characteristic 0 forms part of my doctorial thesis written in 1966 under Professor A. Seidenberg in the University of California at Berkeley and was partially supported by NSF under GP3990. 



\section{PACIFIC JOURNAL OF MATHEMATICS}

\section{EDITORS}

H. SAMELson

Stanford University

Stanford, California 94305

R. R. PHelPS

University of Washington

Seattle, Washington 98105
J. Dugundu

Department of Mathematics

University of Southern Californle

Los Angeles, California 9.0007

RICHARD ARENS

University of California

Los Angeles, California 9.0024

\section{ASSOCIATE EDITORS}
E. F. BECKENBACH
B. H. NeumanN
F. WOLE
K. Yoshida

\section{SUPPORTING INSTITUTIONS}

UNIVERSITY OF BRITISH COLUMBIA

CALIFORNIA INSTITUTE OF TECHNOLOGY

UNIVERSITY OF CALIFORNIA

MONTANA STATE UNIVERSITY

UNIVERSITY OF NEVADA

NEW MEXICO STATE UNIVERSITY

OREGON STATE UNIVERSITY

UNIVERSITY OF OREGON

OSAKA UNIVERSITY

UNIVERSITY OF SOUTHERN CALIFORNIA

\author{
STANFORD UNIVERSITY \\ UNIVERSITY OF TOKYO \\ UNIVERSITY OF UTAH \\ WASHINGTON STATE UNIVERSITY \\ UNIVERSITY OF WASHINGTON \\ AMERICAN MATHEMATICAL SOCIETY \\ CHEVRON RESEARCH CORPORATION \\ NAVAL WEAPONS CENTER
}

The Supporting Institutions listed above contribute to the cost of publication of this Journal, but they are not owners or publishers and have no responsibility for its content or policies.

Mathematical papers intended for publication in the Pacific Journal of Mathematics should be in typed form or offset-reproduced, (not dittoed), double spaced with large margins. Underline Greek letters in red, German in green, and script in blue. The first paragraph or two must be capable of being used separately as a synopsis of the entire paper. The editorial "we" must not be used in the synopsis, and items of the bibliography should not be cited there unless absolutely necessary, in which case they must be identified by author and Journal, rather than by item number. Manuscripts, in duplicate if possible, may be sent to any one of the four editors. Please classify according to the scheme of Math. Rev. Index to Vol. 39. All other communications to the editors should be addressed to the managing editor, Richard Arens, University of California, Los Angeles, California, 90024.

50 reprints are provided free for each article; additional copies may be obtained at cost in multiples of 50 .

The Pacific Journal of Mathematics is published monthly. Effective with Volume 16 the price per volume (3 numbers) is $\$ 8.00$; single issues, $\$ 3.00$. Special price for current issues to individual faculty members of supporting institutions and to individual members of the American Mathematical Society: $\$ 4.00$ per volume; single issues $\$ 1.50$. Back numbers are available.

Subscriptions, orders for back numbers, and changes of address should be sent to Pacific Journal of Mathematics, 103 Highland Boulevard, Berkeley, California, 94708.

PUBLISHED BY PACIFIC JOURNAL OF MATHEMATICS, A NON-PROFIT CORPORATION

Printed at Kokusai Bunken Insatsusha (International Academic Printing Co., Ltd.), 7-17, Fujimi 2-chome, Chiyoda-ku, Tokyo, Japan. 


\section{Pacific Journal of Mathematics}

\section{Vol. 36, No. 2 December, 1971}

George E. Andrews, On a partition problem of H. L. Alder ............ 279

Thomas Craig Brown, An interesting combinatorial method in the theory of locally finite semigroups .......................... 285

Yuen-Kwok Chan, A constructive proof of Sard's theorem ............. 291

Charles Vernon Coffman, Spectral theory of monotone Hammerstein

operators...................................... 303

Edward Dewey Davis, Regular sequences and minimal bases .......... 323

Israel (Yitzchak) Nathan Herstein and Lance W. Small, Regular elements in

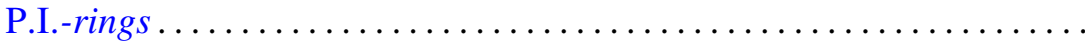

Marcel Herzog, Intersections of nilpotent Hall subgroups ..............

W. N. Hudson, Volterra transformations of the Wiener measure on the space

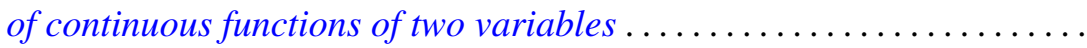

J. H. V. Hunt, An n-arc theorem for Peano spaces ................ 351

Arnold Joseph Insel, A decomposition theorem for topological group

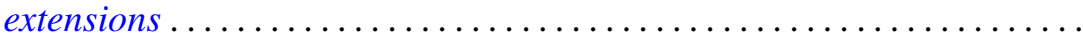

Caulton Lee Irwin, Inverting operators for singular boundary value

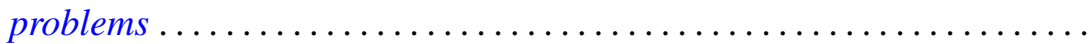

Abraham A. Klein, Matrix rings of finite degree of nilpotency ............ 387

Wei-Eihn Kuan, On the hyperplane section through a rational point of an algebraic variety...

John Hathway Lindsey, II, On a six-dimensional projective representation of $\mathrm{PSU}_{4}(3)$

Jorge Martinez, Approximation by archimedean lattice cones ...

J. F. McClendon, On stable fiber space obstructions .........

Mitsuru Nakai and Leo Sario, Behavior of Green lines at the Kuramochi boundary of a Riemann surface ....................

Donald Steven Passman, Linear identities in group rings. I. .

Donald Steven Passman, Linear identities in group rings. II ...

David S. Promislow, The Kakutani theorem for tensor products of

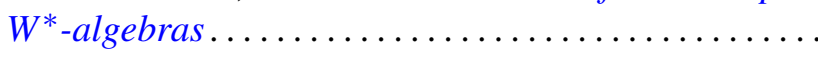

Richard Lewis Roth, On the conjugating representation of a finite group

Bert Alan Taylor, On weighted polynomial approximation of entire functions...

William Charles Waterhouse, Divisor classes in pseudo Galois

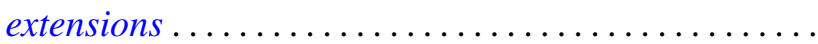

Chi Song Wong, Subadditive functions ...

Ta-Sun $\mathrm{Wu}$, A note on the minimality of certain bitransformation groups 